\title{
Effects of forced-choice runway variations on rats' T-maze serial pattern learning
}

\author{
IZABELA SZELEST and JEROME COHEN \\ University of Windsor, Windsor, Ontario, Canada
}

\begin{abstract}
Rats learned an ordered RNR/RNN serial pattern task in a T-maze where they were shifted to a different runway on Trial 3 only in the RNR series (shift-win/stay-lose group) or only in the RNN series (stay-win/shift-lose group). The shift-win/stay-lose group developed faster speeds on Trial 3 of the RNR than on Trial 3 of the RNN series more easily than the stay-win/shift-lose group. This difference occurred whether all rats were forced onto the same runway on the first two trials (Experiment 1) or onto a different runway on Trial 2 from that on Trial 1 in each series (Experiment 2). Posttraining probe tests revealed that the shift-win/stay-lose group in each experiment relied on the runway shift event in Trial 3 or on the series position to anticipate the second reward within a series. Such reward expectancies were greater when the runway shift occurred in the same series position as during training. These probe tests revealed that the stay-win/shift-lose group relied only on the series position in Experiment 2. Our findings do not support predictions based on an associative predictive validity model. Rather, they reflect rats' predisposition to spontaneously alternate choices in the T-maze, a tendency corresponding to their inherent win-shift foraging strategy. Rats in each group also reduced their speeds less on the nonrewarded Trial 2 when it preceded a rewarded rather than a nonrewarded Trial 3. This effect suggests that rats were able to determine which series contained a second rewarded trial. We discuss the theoretical implications of this Trial 2 speed effect in terms of rats' uncertainty about where this second rewarded trial might occur in the RNR series.
\end{abstract}

Rats eventually run faster down a baited runway than down a nonbaited runway when repeatedly exposed to a fixed sequence of massed rewarded $(\mathrm{R})$ and nonrewarded (N) trials (Capaldi, Verry, Nawrocki, \& Miller, 1984; Hulse, 1978). Such differential responding indicates that rats learn to accurately anticipate when a trial in such a series will be rewarded. Rats acquire such serial pattern performance more easily when each series is separated by long intervals or alternates between visually distinct runways (Fountain, Henne, \& Hulse, 1984; Haggbloom, 1993; Haggbloom, Birmingham, \& Scranton, 1992). Furthermore, rats can acquire accurate serial pattern performance for different outcome sequences, such as RNN and RNR series, when each series occurs on a different runway (Burns, Dunkman, \& Detloff, 1999) or in a fixed order within each session (Capaldi, Nawrocki, \& Verry, 1983). In either preparation, rats not only develop faster speeds on Trial 3 of the RNR than on Trial 3 of the RNN series but also reduce their speeds less on Trial 2 of the

The present study was supported by a grant to the second author from the Natural Sciences and Engineering Research Council of Canada. Experiment 1 served as a thesis for the first author in partial fulfillment of a B.Sc. in Biology and Psychology (Behaviour, Cognition, \& Neuroscience program) at the University of Windsor. Portions of this research were originally presented at the Tri-State Conference on Animal Learning, Kent State University (May 2004). The authors thank R. Burns, S. Fountain, and E. J. Capaldi for their helpful comments on the manuscript. Request reprints from J. Cohen, Department of Psychology, University of Windsor, Windsor, ON, N9B 3P4 Canada (e-mail: jcohen@uwindsor.ca).
RNR than on Trial 2 of the RNN series, the Trial 2 speed effect. These effects demonstrate that rats have learned which series contains a second rewarded trial.

Serial pattern learning of ordered RNR/RNR series (Capaldi et al., 1983) may be analogous to how humans chunk verbal items from long lists to improve their immediate recall (Miller, 1956; Simon, 1974). For example, the letters D E A F B I C I A are easier to recall in order when they are spatially or temporally grouped (or chunked) into abbreviations for three U.S. law enforcement and intelligence agencies, DEA FBI CIA. Rats' serial pattern learning of ordered RNN/RNR series could represent their integration of individual series chunks into a higher level list chunk (Capaldi, 1992). Although human chunking involves the use of abstract categories or a global rule, that of rats may reflect only acquisition and memory of sequential associations among various events (Capaldi \& Molina, 1979; Capaldi, Nawrocki, Miller, \& Verry, 1986). An examination of ordered RNR/RNN serial pattern learning based on sequential associations follows.

In learning an ordered RNR/RNN serial pattern, rats may learn that a rewarded trial always follows a longer interval outside the runway and that a nonrewarded trial always immediately follows a rewarded trial. Both a nonrewarded and a rewarded third trial, however, immediately occur after an RN sequence of trials. Consequently, this RN sequence does not provide as consistent an association with either Trial 3 outcome as does a longer interval for a Trial 1 outcome or as does Trial 1's outcome for that of Trial 2. Thus, the associative predictive validity of the 
RN sequence for a final rewarded trial would be only .50 . Only the position of the RNR series in a session is a better predictor for this outcome. Rats might learn to associate different preseries intervals with each series. Suppose, for example, a rat always experiences an RNR series before an RNN series in every session. It might learn to determine the position of each series by learning that a longer interval of several hours in the home cage in the colony room always occurs before the RNR series, whereas a shorter interval of 15-20 min in a holding cage near the runway always occurs before the RNN series. If a rat always experienced an RNN series before an RNR series, it would learn that the longer interval outside the runway signaled the RNN series and the shorter interval outside the runway signaled the RNR interval. Thus an RNR position cued by its preceding interval would have a predictive validity of 1.00 for a Trial 3 reward.

Capaldi, Birmingham, and Miller (1999, Experiment 2) investigated the effects of varying runways on rats' performance of an ordered RNR/RNN series task. They accounted for their findings with an associative predictive validity model. In that experiment, all rats ran on a white or black runway over the first two trials within each series. Some rats were shifted onto the opposite runway (from white to black or from black to white) on the last trial in each series. For the purposes of the present study, we designated these rats as the shift-win/shift-lose group, where the first term refers to the runway event and outcome on Trial 3 of the RNR series, and the second term, to those events on Trial 3 of the RNN series. The other rats in that experiment were shifted onto the opposite runway on Trial 3 only in the RNR series and therefore were designated as the shift-win/stay-lose group. The shift-win/ stay-lose group ran consistently faster on the last trial of the RNR series than on the last trial of the RNN series and reduced their speeds more on the always nonrewarded Trial 2 in either series more than did the shift-win/shiftlose group. The latter group never developed reliably faster speeds on Trial 3 in the RNR series than on Trial 3 in the RNN series. Note also that rats in the earlier study $(\mathrm{Ca}-$ paldi et al., 1983) were exposed to only one runway and also anticipated the final rewarded trial more accurately than the shift-win/shift-lose group. Unlike rats in the earlier study, however, neither group reduced its speeds on Trial 2 less in the RNR series than in the RNN series.

According to Capaldi et al. (1999), these group differences resulted from Trial 3's runway shift in the RNR series having greater predictive validity for that outcome in the shift-win/stay-lose condition than in the shift-win/ shift-lose condition (1.00 vs. .50). The predictive validity of the RN sequence for Trial 3 outcomes was .50 in either group and thus became overshadowed by the runway shift event only in the shift-win/stay-lose group. This explanation did not take into account any possible influence of a highly predictive position of the RNR series in each group. Their shift-win/stay-lose group could have learned the serial pattern task by attending to either highly predictive runway shift or RNR series position cues or both and by ignoring the less predictive $\mathrm{RN}$ sequence. The shift-win/shift-win group would have had to have learned to ignore both equally less predictive RN sequence and runway shift events and attend to RNR position, however. This might explain why the rats exposed to only one runway in Capaldi et al. (1983) also performed better than the shift-win/shift-lose group. That is, these rats also had only to learn to ignore $\mathrm{RN}$ sequences in favor of the more valid RNR position. Perhaps rats in the shift-win/shiftlose group might eventually have learned to use their more valid RNR position with more training.

The question remains whether the shift-win/stay-lose rats learned to anticipate a final rewarded trial only from a runway shift event or also from their equally highly predictive RNR series position. That is, did these rats merely learn to wait for a shift in runways to anticipate a subsequent reward in a series? Or did they use both a runway shift and RNR position? If they used both sources of information, did they use them independently or integrate them in some way? For example, did they learn to anticipate a final rewarded trial only when a runway shift event occurred at one series position? Perhaps these rats used the runway shift event only as another redundant series position marker. Accordingly, rats would have performed as well whether they received the runway shift event on Trial 3 only in the RNN series (a stay-win/shift-lose group) or only in the RNR series (the shift-win/stay-lose group). If the predictive validity of a runway event for a Trial 3 reward is important, this hypothetical stay-win/ shift-lose group would also perform more poorly than the shift-win/stay-lose group. This is because the predictive validity of a runway stay event for a reward is only .33 for this group; there are two other nonrewarded runway stay events (from Trial 1 to Trial 2 in each series). Therefore, the stay-win/shift-lose group would also have to learn to ignore an even less predictive runway stay event as well as the $\mathrm{RN}$ sequence in favor of the most predictive position of the RNR series.

Even if the shift-win/stay-lose group performed better than a proposed stay-win/shift-lose group on the ordered RNR/RNN serial pattern task, the question remains how each group learned to anticipate a reward on Trial 3. One would have to systematically vary the order of the series and Trial 3's runway event on posttraining test probe sessions to answer this question. One possibility is that rats in the shift-win/stay-lose group simply learned to wait for a runway shift to expect a subsequent rewarded trial in a series (the runway event only hypothesis, RE only). The RE-only hypothesis predicts that they should run equally fast on Trial 3 on any series containing the runway shift trial. Another possibility is that these rats used both series position and runway shift as separate predictors (the runway event or series position hypothesis, RE or SP). The RE or SP hypothesis predicts that they should run fastest when the runway shift event has occurred in the original series position but not quite as fast when it has occurred in the other series position. The third possibility is that these rats had learned only whether a rewarded runway shift had occurred in the first or in the second series of trials (runway event at series position hypothesis, $\mathrm{RE}$ at $\mathrm{SP}$ ). The RE 
at SP hypothesis predicts that they should run fast only when they encounter the runway shift in original series position. As already suggested, a proposed stay-win/shiftlose group would learn to use RNR position only during training (series position only hypothesis, SP only). The SP-only hypothesis predicts that rats should run fastest only on the last trial of the series occurring in the original position regardless of its runway event. In other words, the original position of the RNR series would overshadow the runway stay event on Trial 3 in a stay-win/shift-lose group but not the runway shift event on Trial 3 in the shiftwin/stay-lose group.

We note that other studies have successfully employed similar probe test procedures to determine relative dominance of redundant cues in multiple-series serial pattern tasks (Burns et al., 1999; Cohen, Simpson, Westlake, \& Hamelin, 2002, Experiment 2). For example, Burns et al. (1999) found that runway floor texture overshadowed food type (cereal and food pellets) for cuing RNN and RNR series. Cohen et al. (2002, Experiment 2) found that Trial 2's outcome was dominant but did not overshadow a runway cue for anticipating Trial 3 outcomes in RNR and RRN series.

Therefore, we conducted the present study as a modified replication of Capaldi et al. (1999, Experiment 2). Our study consisted of two experiments. In Experiment 1, we trained rats on an ordered RNR/RNN series task under either a shift-win/stay-lose or a stay-win/shift-lose condition but not under a shift-win/shift-lose condition. In our task, the two runways consisted of a left black and a right striped side runway in a T-maze. Rats in both groups ran down one of the side runways on the first two trials of each series. The shift-win/stay-lose group ran down the opposite side runway on Trial 3 of the RNR series and down the same runway on Trial 3 as on the first two trials of the RNN series. The stay-win/shift-lose group ran down the same side runway on Trial 3 of the RNR series and down the opposite side runway on Trial 3 of the RNN series. Our rats received far more training than did the rats in Capaldi et al. (1999) (72 vs. 28 sessions). Following training, we also gave our rats different types of probe test sessions to test the various hypotheses concerning how rats learned to determine Trial 3 outcomes.

According to the associative predictive validity model, the shift-win/stay-lose group should develop accurate serial pattern performance more quickly than the stay-win/ shift-lose group. We expected probe tests to show that the position of the RNR series had overshadowed the rewarded runway stay event in the stay-win/shift-lose group but not the rewarded runway shift event in the shift-win/shift-lose group. More importantly, we expected probe test sessions to reveal more precisely how the shift-win/stay-lose rats used the runway shift event and the position of the RNR series to anticipate the second rewarded trial in a series.

In Experiment 2, we further tested the associative predictive validity model. We reversed the predictive validity of each group's rewarded runway event on Trial 3 by forcing rats down the opposite runway on Trial 2 from that on Trial 1 of each series. The new shift-win/stay-lose group still received a rewarded third trial only by being forced down the opposite runway on Trial 3 from that on Trial 2. The new stay-win/shift-lose group still received a rewarded third trial only by being forced down the same runway on Trial 3 as on Trial 2. From this change, the predictive validity of a rewarded runway shift event in the shift-win/stay-lose group was reduced to .33 from the 1.00 of Experiment 1 . The predictive validity of a rewarded runway stay event in the stay-win/shift-lose group was now increased to 1.00 from the .33 of Experiment 1 . By reversing each runway event's predictive validity, we expected to reverse the effects found in Experiment 1. That is, the reconstituted shift-win/stay-lose group should develop accurate serial pattern performance more slowly than the stay-win/shift-lose group. The position of the RNR series should also overshadow the rewarded runway shift event in the shift-win/stay-lose group but not the rewarded runway stay event in the stay-win/shift-lose group. Moreover, the stay-win/shift-lose group should employ its now highly relevant runway stay event in the same way as the shift-win/stay-lose group in Experiment 1 had used its Trial 3 runway shift event.

\section{EXPERIMENT 1}

We have discussed the rationale, basic design, and general predictions for this experiment. Here, we describe its design and the possible patterns of performance in more detail. As already noted, our rats received considerably more training on their ordered serial pattern learning task than did rats in prior studies ( 72 vs. 28 sessions in Capaldi et al., 1983; 32 sessions in Capaldi et al., 1999, Experiment 2). They also received a second test phase, consisting of alternating test probe and regular training sessions. The rats received extensive training to ensure that both groups eventually developed similar serial pattern performance that they could maintain over the alternating training sessions during the test phase. Therefore, although we analyzed each trial's speeds during training to determine acquisition differences between groups, we focused on Trial 3's speeds of each series during the test phase. Thus the specific patterns of performance that could occur during the test probe sessions were based on predicted Trial 3 speed differences between test probes and their accompanying training sessions.

Table 1 illustrates the different types of training and probe test sessions given to the shift-win/stay-lose and stay-win/shift-lose groups. Although the order of each series was equally counterbalanced within each group as in Table 2, because of limitations of space we illustrate examples based on one fixed order of series during training in Table 1. As can be seen in our example, the shiftwin/stay-lose group receive an $\mathrm{RN} \mid \mathbf{R}-\mathrm{RNN}$ sequence and the stay-win/shift-lose group receive an RNR-RN|N sequence, where "-_" indicates the interseries interval in a session and "|" represents a runway shift. We used this form of notation to further depict the three types of probe tests that each group experienced following training. The series outcomes in these probe tests are in italics to dis- 
Table 1

\begin{tabular}{|c|c|c|c|}
\hline & \multicolumn{3}{|c|}{ Probe Test } \\
\hline & SO-r/RE-m & SO-m/RE-r & SO-r/RE-r \\
\hline \multicolumn{4}{|c|}{ Shift-Win/Stay-Lose Group } \\
\hline Training series & $\mathrm{RN} \mid \mathbf{R}-\mathrm{RNN}$ & $\mathrm{RN} \mid \mathbf{R}-\mathrm{RNN}$ & $\mathrm{RN} \mid \mathbf{R}-\mathrm{RNN}$ \\
\hline Test series & $R N N-R N \boldsymbol{R}$ & $R N \boldsymbol{R}-R N \mid \boldsymbol{N}$ & $R N \mid \boldsymbol{N}-R N \boldsymbol{R}$ \\
\hline \multicolumn{4}{|l|}{ Hypothesis } \\
\hline Runway shift only & $|\boldsymbol{R}=| \mathbf{R} N=\mathbf{N}$ & $\boldsymbol{R}<|\mathbf{R}| \mathbf{N}>\mathbf{N}$ & $\boldsymbol{R}<|\mathbf{R}| \mathbf{N}>\mathbf{N}$ \\
\hline Runway shift or trained RNR series position & $\boldsymbol{R}<\mid \mathbf{R} N>\mathbf{N}$ & $\boldsymbol{R}<|\mathbf{R}| \boldsymbol{N}>\mathbf{N}$ & $\boldsymbol{R}<|\mathbf{R}| \boldsymbol{N}>\mathbf{N}$ \\
\hline Runway shift at trained RNR series position & $|\boldsymbol{R}<| \mathbf{R} \mid \boldsymbol{N}=\mathbf{N}$ & $\boldsymbol{R}<|\mathbf{R}| \boldsymbol{N}=\mathbf{N}$ & $\boldsymbol{R}<|\mathbf{R}| \boldsymbol{N}>\mathbf{N}$ \\
\hline \multicolumn{4}{|c|}{ Stay-Win/Shift-Lose Group } \\
\hline Training series & RNR-RN|N & RNR-RN|N & RNR-RN|N \\
\hline Test series & $R N \mid \boldsymbol{N}-R N \boldsymbol{R}$ & $R N \boldsymbol{R}-R N N$ & $R N N-R N \mid \boldsymbol{R}$ \\
\hline \multicolumn{4}{|l|}{ Hypothesis } \\
\hline Trained RNR position & $\boldsymbol{R}<\mathbf{R}|\boldsymbol{N}>| \mathbf{N}$ & $\boldsymbol{R}=\mathbf{R} \boldsymbol{N}=\mid \mathbf{N}$ & $|\boldsymbol{R}<\mathbf{R} N>| \mathbf{N}$ \\
\hline
\end{tabular}

tinguish them from those in their accompanying training sessions.

Three different types of probe test sessions alternated with regular training sessions. In one type of probe test, we reversed the series order but maintained the same Trial 3 runway event in each series (series order reversed/runway event maintained: SO-r/RE-m). Thus in our example, a shift-win/stay-lose rat received an $R N \boldsymbol{N}-R N \mid \boldsymbol{R}$ sequence (changed from an RN|R-RNN sequence) and a stay-win/ shift-lose rat received an $R N \mid \boldsymbol{N}-R N \boldsymbol{R}$ sequence (changed from an RNR-RN|N sequence). On a second type of probe test, we maintained the original series order but reversed its Trial 3 runway event (series order maintained/runway event reversed: SO-m/RE-r). Thus, a shift-win/stay-lose rat now received an $R N R-R N \mid N$ sequence (changed from an RN|R-RNN sequence) and a stay-win/shift-lose rat received an $R N \mid \boldsymbol{R}-R N N$ sequence (changed from an $\mathrm{RNR}-\mathrm{RN} \mid \mathbf{N}$ sequence). In the third type of probe test, we reversed both series order and each series' Trial 3 runway event (series order reversed/runway event reversed: SO-r/ RE-r). Thus, a shift-win/stay-lose rat received an $R N \mid N$ $R N \boldsymbol{R}$ sequence (changed from an $\mathrm{RN} \mid \mathbf{R}-\mathrm{RNN}$ sequence) and a stay-win/shift-lose rat received an $R N \boldsymbol{N}-R N \mid \boldsymbol{R}$ sequence (changed from an RNR-RN|N sequence).

Each of the possible ways in which the shift-win/staylose group might learn to anticipate a Trial 3 reward (RE only; RE or SP; RE at SP) predicts specific reactions on Trial 3 of each series in each probe test. The one way (SP only) in which the stay-win/shift-lose group might learn to anticipate a Trial 3 reward also predicts a specific reaction on Trial 3 of each series in each probe test. In Table 1, we have expressed these predicted effects as differences in Trial 3 speeds between a test probe session and its preceding training session for each series. These effects are based on the assumption that rats will continue to anticipate a reward on Trial 3 in the RNR series (run faster) than

Table 2

Sequences of Left $(\leftarrow)$ and Right $(\rightarrow)$ Forced Choice Patterns for Each Group During Training in Each Experiment

\begin{tabular}{|c|c|c|c|c|}
\hline \multicolumn{5}{|c|}{ Experiment 1} \\
\hline Shift-win/stay-lose & \multicolumn{2}{|c|}{$\mathrm{RN} \mid \mathrm{R}-\mathrm{RNN}$} & \multicolumn{2}{|c|}{ RNN-RN|R } \\
\hline Stay-win/shift-lose & \multicolumn{2}{|c|}{$\mathrm{RN} \mid \mathrm{N}-\mathrm{RNR}$} & \multicolumn{2}{|c|}{$\mathrm{RNR}-\mathrm{RN} \mid \mathrm{N}$} \\
\hline Sequence A & $\rightarrow \rightarrow \leftarrow$ & $\rightarrow \rightarrow \rightarrow$ & $\rightarrow \rightarrow \rightarrow$ & $\rightarrow \rightarrow \leftarrow$ \\
\hline Sequence B & $\leftarrow \leftarrow \rightarrow$ & $\leftarrow \leftarrow \leftarrow$ & $\leftarrow \leftarrow \leftarrow$ & $\leftarrow \leftarrow \rightarrow$ \\
\hline Sequence $\mathrm{C}$ & $\leftarrow \leftarrow \rightarrow$ & $\rightarrow \rightarrow \rightarrow$ & $\rightarrow \rightarrow \rightarrow$ & $\leftarrow \leftarrow \rightarrow$ \\
\hline Sequence D & $\rightarrow \rightarrow \leftarrow$ & $\leftarrow \leftarrow \leftarrow$ & $\leftarrow \leftarrow \leftarrow$ & $\rightarrow \rightarrow \leftarrow$ \\
\hline \multicolumn{5}{|c|}{ Experiment 2} \\
\hline Shift-win/stay-lose & \multicolumn{2}{|c|}{$\mathrm{R}|\mathrm{N}| \mathrm{R}-\mathrm{R} \mid \mathrm{NN}$} & \multicolumn{2}{|c|}{$\mathrm{R}|\mathrm{NN}-\mathrm{R}| \mathrm{N} \mid \mathrm{R}$} \\
\hline Stay-win/shift-lose & \multicolumn{2}{|c|}{$\mathrm{R}|\mathrm{N}| \mathrm{N}-\mathrm{R} \mid \mathrm{NR}$} & \multicolumn{2}{|c|}{$\mathrm{R}|\mathrm{NR}-\mathrm{R}| \mathrm{N} \mid \mathrm{N}$} \\
\hline Sequence A & $\rightarrow \leftarrow \rightarrow$ & $\rightarrow \leftarrow \leftarrow$ & $\rightarrow \leftarrow \leftarrow$ & $\rightarrow \leftarrow \rightarrow$ \\
\hline Sequence B & $\leftarrow \rightarrow \leftarrow$ & $\leftarrow \rightarrow \rightarrow$ & $\leftarrow \rightarrow \rightarrow$ & $\leftarrow \rightarrow \leftarrow$ \\
\hline Sequence C & $\leftarrow \rightarrow \leftarrow$ & $\rightarrow \leftarrow \leftarrow$ & $\rightarrow \leftarrow \leftarrow$ & $\leftarrow \hookrightarrow \leftarrow$ \\
\hline Sequence D & $\rightarrow \leftarrow \rightarrow$ & $\leftarrow \rightarrow \rightarrow$ & $\leftarrow \rightarrow \rightarrow$ & $\rightarrow \leftarrow \rightarrow$ \\
\hline
\end{tabular}

Note- $\mid \mathrm{N}$ or $\mid \mathrm{R}$ is a shift in runway from that on the previous trial within a series on a nonrewarded or on a rewarded trial, respectively. 
in the RNN series (run slower) in the training sessions in Phase 2. As will be reported later, this assumption was empirically supported.

To elucidate the contents of Table 1, we will explain each of the predicted differences in speed for the shift-win/ stay-lose group and then those for the stay-win/shift-lose group as follows. According to the RE-only hypothesis, the shift-win/stay-lose rats should anticipate a reward only on the $\mid \boldsymbol{R}$ trial during SO-r/RE-r probe tests and only on the $\mid \boldsymbol{N}$ trial during either SO-m/RE-r or SO-r/RE-r probe tests. Therefore, they should run as fast on $\mid \boldsymbol{R}$ and $\mid \mathbf{R}$ trials or as slow on $\boldsymbol{N}$ and $\mathbf{N}$ trials but run slower on $\boldsymbol{R}$ than on $\mid \mathbf{R}$ trials or faster on $\mid \boldsymbol{N}$ than on $\mathbf{N}$ trials $(|\boldsymbol{R}=| \mathbf{R}$ or $\boldsymbol{N}=\mathbf{N}$; $\boldsymbol{R}<\mid \mathbf{R}$ or $\mid \boldsymbol{N}>\mathbf{N})$. According to the RE or SP hypothesis, these rats should anticipate a reward more on the $\mid \boldsymbol{N}$ trial during SO-r/RE-r probe tests than on an $\mid \boldsymbol{R}$ trial during SO-r/RE-m probe tests or on an $\mid \boldsymbol{N}$ trial during SO-m/RE-r probe tests. Therefore, they should not run quite as fast on $\mid \boldsymbol{R}$ or $\boldsymbol{R}$ trials nor quite as slow on $\boldsymbol{N}$ or $\mid \boldsymbol{N}$ trials during SO-r/RE-m or SO-m/RE-r probe tests as on $\mid \mathbf{R}$ or $\mathbf{N}$ trials, respectively $(|\boldsymbol{R}<| \mathbf{R}$ or $\boldsymbol{N}>\mathbf{N} ; \boldsymbol{R}<\mid \mathbf{R}$ or $\mid \boldsymbol{N}>\mathbf{N})$. On the other hand, they should run more slowly on $\boldsymbol{R}$ trials and more quickly on $\mid \boldsymbol{N}$ trials during an SO-r/RE-r probe test than on the $\mid \mathbf{R}$ trials or $\mathbf{N}$ trials, respectively $(\boldsymbol{R}<\mid \mathbf{R}$ or $\mid \boldsymbol{N}>\mathbf{N})$. According to the RE at SP hypothesis, these rats will anticipate a Trial 3 reward only on an $\mid \boldsymbol{N}$ trial during SO-r/RE-r probe tests. Therefore, they should run more slowly on $\boldsymbol{R}$ trials and or more quickly on $\mid \boldsymbol{N}$ trials during SO-r/RE-r probe tests than on $\mid \mathbf{R}$ trials and on $\mathbf{N}$ trials, respectively $(\boldsymbol{R}<\mid \mathbf{R}$ or $\mid \boldsymbol{N}>\mathbf{N})$. However, these rats would not be anticipating a reward on Trial 3 of either series during SO-r/RE-m or SO-m/RE-r probe tests. Consequently, they should run as slowly on $\boldsymbol{N}$ and $\mid \boldsymbol{N}$ trials as on $\mathbf{N}$ trials and more slowly on $\mid \boldsymbol{R}$ or $\boldsymbol{R}$ trials than on $\mid \mathbf{R}$ trials $(\mid \boldsymbol{R}<$ $\mid \mathbf{R}$ and $\boldsymbol{N}=\mathbf{N} ; \boldsymbol{R}<\mid \mathbf{R}$ and $\mid \boldsymbol{N}=\mathbf{N})$.

The stay-win/shift-lose rats were expected to use only the RNR position to anticipate the Trial 3 reward. Therefore, they should anticipate a reward only on an $\mid \boldsymbol{N}$ trial during SO-r/RE-m test probes, on an $\mid \boldsymbol{R}$ trial during SO-m/ RE-r test probes, and on an $\boldsymbol{N}$ trial during SO-r/RE-r test probes. Consequently, they should run slower on $\boldsymbol{R}$ trials and faster on $\mid \boldsymbol{N}$ trials during SO-r/RE-r probe tests than on $\mathbf{R}$ trials and $\mid \mathbf{N}$ trials, respectively $(\boldsymbol{R}<\mathbf{R}$ or $|\boldsymbol{N}>| \mathbf{N})$. However, they should run equally fast and slow on $\mid \boldsymbol{R}$ and $\boldsymbol{N}$ trials during SO-m/RE-r probe tests as on R and $\mid \mathbf{N}$ trials, respectively $(\mid \boldsymbol{R}=\mathbf{R}$ and $\boldsymbol{N}=\mid \mathbf{N})$. They should also run slower on $\mid \boldsymbol{R}$ trials and faster on $\boldsymbol{N}$ trials during the SO-r/RE-r probe tests than on $\mathbf{R}$ trials or $\mid \mathbf{N}$ trials, respectively $(\mid \boldsymbol{R}<\mathbf{R}$ or $\boldsymbol{N}>\mid \mathbf{N})$.

\footnotetext{
Method

Subjects

Eight male Long-Evans hooded rats originally from the Charles River Breeding Farms, St. Constant, QC, served in this experiment. Veterans of a previous two-choice object discrimination experiment, they were 12 months old, and they weighed over $450 \mathrm{~g}$ at the start of this study. We employed the general maintenance and housing conditions described in Cohen, Westlake, and Pepin (2001). That is, we gave each rat its daily ration of 20-25 g of Purina Rodent Chow in its individual holding cage for $2 \mathrm{~h}$ following each experimental
}

session, and we continuously provided water in individual holding and group home cages. This regimen maintained the rats at about $90 \%$ of their free-feeding weight.

\section{Apparatus}

We used an enclosed T-maze from a previous study (see Cohen et al., 2001, Figure 1, p. 414). It consisted of long left black and right striped runways that extended from a short center alley. A return alley, located $20 \mathrm{~cm}$ from the beginning of each side runway, curved back to the center alley's start chamber. The distance from the entrance to the center arm to goal area at the end of each side runway was $190 \mathrm{~cm}$. A moveable $10 \times 12 \mathrm{~cm}$ food tray, containing eight food wells, could be slid into the end of each goal area. The experimenter baited each well with a shelled roasted unsalted sunflower seed (eight seeds in each tray). A 40-W light over each side runway and a $40-\mathrm{W}$ light behind a translucent plastic panel at the end of the center alley illuminated the maze. The experimenter activated a silent digital clock by lifting the start chamber's guillotine door to the center arm when the rat faced it. The clock stopped when the rat intersected a photobeam at the beginning of the goal area at the end of the runway. The rat could return to the start chamber through the return alley when the experimenter opened a sliding door on the sidewall of the start chamber.

\section{Procedure}

Training phase. Prior to randomly dividing rats into the two equal training groups, we shaped them to run down each runway for sunflower seeds as described in Cohen, Westlake, and Szelest (2004). The rats in each group were trained for 72 sessions on RNR and RNN series that occurred in a fixed order within each session. Series order was counterbalanced equally over rats within each group. The rats received 10 -sec intertrial intervals within each series in the start chamber of the T-maze and a 15- to 20-min interval between the first and second series in their individual holding cages outside the apparatus. As already described, the rats in the shift-win/stay-lose group were always forced down the same runway on all three trials in the RNN series and forced down the opposite runway on Trial 3 in the RNR series. The rats in the stay-win/shift-lose group received a change in runway on Trial 3 only in the RNN series. As is shown in Table 2, these runway variations generated four different sequences. Each sequence occurred equally over training in a semirandom fashion without occurring on more than two sessions in a row.

Test phase. Following training, the rats received a test phase of 48 sessions of alternating even numbered probe test and odd numbered regular training sessions. Table 1 illustrates the four possible sequences of each of the three types of test probes: SO-r/RE-m, SO-m/RE-r, and SO-r/RE-m. Each type of probe test occurred eight times and was randomly distributed over the even numbered trials. No type of probe test occurred on more than two even numbered sessions in a row.

Dependent measures. We recorded a rat's running time on each trial to the nearest $0.1 \mathrm{sec}$ and transformed it into a speed score (in centimeters/second). If a rat refused to leave the center alley for $100 \mathrm{sec}$ or ran into and stayed in the return alley for $10 \mathrm{sec}$ without going to the end of the side runway, it was allowed back into the start chamber. We recorded the running speed on such an occasional bypass response as a default speed of $2 \mathrm{~cm} / \mathrm{sec}$. We calculated each rat's average speed for each trial over successive blocks of eight sessions during training. For the test phase, we averaged each trial's speeds over the eight sessions of each probe test and over its eight preceding training sessions. We calculated the speed difference scores from these two sets of trials. For example, if a rat received an SO-r/RE-m probe test on Sessions 2, 10, 18, 24, 36, 40, 42, and 48, we averaged its speeds also on trials over the preceding Training Sessions 1, 9, 17, $23,35,39,41$, and 47 . We then calculated a difference score between the averaged even numbered and odd numbered sessions' speeds for each trial in each series. Thus the use of difference scores reduced between-subjects variance and increased the power of our statistical 


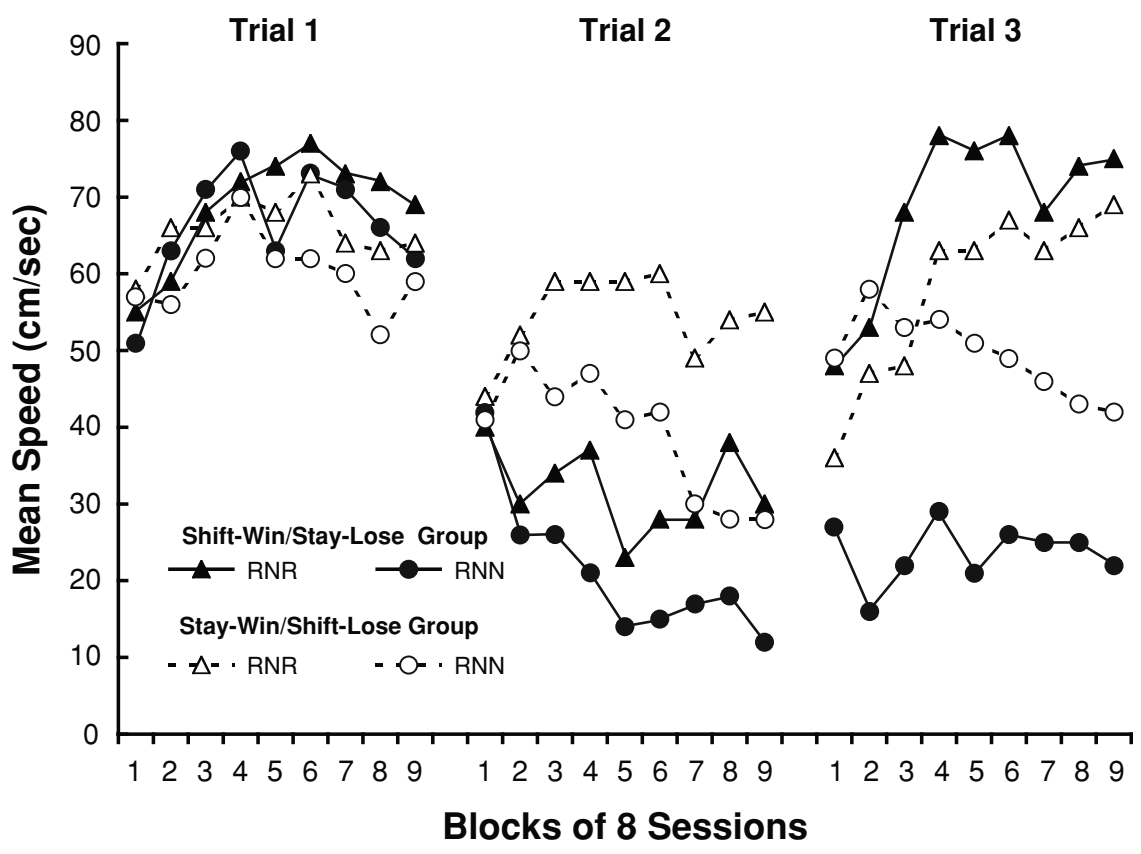

Figure 1. Mean speeds on each trial in each series over blocks of eight sessions for each group during the training phase in Experiment 1.

analysis of test phase performance. The results from ANOVAs from these averaged speed scores on each trial in the training phase and the difference scores in the test phase were considered significant at $p<.05$.

\section{Results and Discussion}

\section{Training phase}

Figure 1 shows each trial's mean speed in each series over successive blocks of eight sessions for each group with series order collapsed. Standard errors of the mean (not shown) ranged from \pm 4 to $\pm 8 \mathrm{~cm} / \mathrm{sec}$ in this and the training phase of the subsequent experiment. Both groups developed higher speeds on $\mathrm{R}$ trials than on $\mathrm{N}$ trials. The shift-win/stay-lose group, however, reduced its speeds on Trial 2 in each series and on Trial 3 in the RNN series more than the stay-win/shift-lose group. Indeed, the staywin/shift-lose group failed to run significantly slower on Trial 2 than on Trial 1 in the RNR series until the last block of that series $[F(1,3)=21.06]$. Separate three-way (group $\times$ series $\times$ block) ANOVAs for each trial confirmed our observation that speeds on Trials 2 and 3 but not on Trial 1 were a function of both group and series. As can be seen in the left portion of Figure 1, and as was supported by a significant effect only for blocks of sessions $[F(8,48)=6.50]$, both groups similarly increased their Trial 1 speeds over the first four blocks. As can be seen in the middle of the figure, on Trial 2, the shift-win/staylose group reduced its speeds more rapidly and to lower levels than did the stay-win/shift-lose group. A significant effect of group $[F(1,6)=24.37]$ and a significant interaction of group $\times$ block $[F(8,48)=4.06]$ supported this observation. However, as is evident and supported by a significant series effect $[F(8,48)=5.71]$, each group reduced its Trial 2 speeds more in the RNN than in the RNR series. As can be seen in the right portion of Figure 1, the shift-win/stay-lose group developed higher Trial 3 speeds in the RNR than in the RNN series from the first block of sessions. The stay-win/shift-lose group developed similar differences only from the fourth block on and did not reduce its Trial 3 speeds in the RNN series to levels achieved by the shift-win/stay-lose group. A significant triple interaction $[F(8,48)=2.16]$ and a group $\times$ series interaction on the last block $[F(1,6)=7.27]$ supported these observations.

As in Capaldi et al. (1999, Experiment 2), our shiftwin/stay-lose group developed greater and more consistent differences in Trial 3 speeds between the RNR and RNN series and reduced its Trial 2 speeds in each series more than the other group. Two differences between these experiments stand out, however. First, our stay-win/shiftlose group also developed differences in speeds on Trial 3 as a function of its outcome that were similar to but not as great as those seen in our shift-win/stay-lose group. The stay-win/shift-lose group began to show reliable anticipation for a rewarded final trial in their RNR series only after 24 training sessions. Perhaps the shift-win/shift-lose group in Capaldi et al. (1999) would have also developed similar speed differences had it been given more than 28 training sessions. Second, our rats in each group reduced their speeds less on Trial 2 when it preceded a final rewarded trial than when it preceded a final nonrewarded trial. This Trial 2 speed effect was noticeably absent in Capaldi et al. (1999, Experiment 2), but it replicates that found in Capaldi et al. (1983). Perhaps rats in the shift- 
win/stay-lose group, if not those in the shift-win/shift-lose group, in Capaldi et al. (1999), might have developed a similar effect with continued training.

\section{Test Phase}

Prior to analyzing differences in speed scores within this phase, we examined differences in speeds between the last block of the training phase and training sessions in this phase. Rats in each group ran equally fast on their final rewarded trials and equally slow on their final nonrewarded trials over phases. No significant differences were uncovered. Therefore, each group continued to anticipate a final rewarded trial during the training sessions in the test phase just as they had by the end of their training phase. Also not surprisingly, we did not detect any differences in speeds between training and test probe sessions on Trials 1 or 2 in each series. Therefore, we present only data that reflect rats' anticipation of a reward on Trial 3 as a function of its runway event and series position. For ease of explication, we depict mean Trial 3 speeds for each type of test probe and its accompanying training sessions for each series separately for each group (Figure 2A, shift-win/stay-lose group; Figure 2B, stay-win/shift-lose group). We did not use different notations for the training and probe test series as in Table 1, except to label each Trial 3 outcome bar. Rather, we refer to differences in Trial 3 speeds between the training and test RNR series and between the training and test RNN series for each type of probe test.

Before analyzing each group separately, we directly compared them in a 2 (groups) $\times 2$ (series) $\times 3$ (test probe type) ANOVA. A comparison of Figures $2 \mathrm{~A}$ and $2 \mathrm{~B}$ reveals that the shift-win/stay-lose trained rats decreased their Trial 3 speeds in the RNR series and increased them in the RNN series more than the stay-win/shift-lose trained rats when series order was maintained but runway events were reversed or when both factors were reversed (SO-m/RE-r and SO-r/RE-r probe tests). Each group showed similar speed changes, however, when series order was reversed but runway events were maintained (SO-r/RE-m test probes). The significant effect of group $[F(1,8)=8.42]$ and the significant group $\times$ test probe type interaction $[F(2,12)=6.60]$ supported these observations. The following analysis of each group was carried out by separate 2 (series) $\times 3$ (test probe types) ANOVAs.

\section{Shift-Win/Stay-Lose Group}

Every rat reduced its speed on Trial 3 in the RNR series and increased it in the RNN series during each type of probe test. As can be seen in Figure 2A, however, these changes were greatest when both series order and runway events were reversed (SO-r/RE-r probe tests) and least when series order was reversed but runway events were maintained (SO-r/RE-m test probes). A significant probe test effect $[F(2,6)=9.02]$ and significant pairwise comparisons between SO-r/RE-r and SO-m/RE-r or SO-r/RE-m probe tests $\left[F_{\mathrm{S}}(1,3)=15.65 ; 15.39\right]$ supported this observation. These rats' apparent greater changes in speed in the SO-m/RE-r probe tests than in the SO-r/RE-m probe tests were not significant $[F(1,3)=3.87, p=.14]$. We note that these rats appeared to decrease their Trial 3 speeds in the RNR series as much as they increased them in the RNN series during test probe sessions. An $F<1$ for an interaction between outcome and test session supported this observation. These results show that a runway shift event did not merely overshadow RNR position for anticipating a reward on Trial 3 in these rats. Rather, they had also learned whether a runway shift always occurred in the first or second series of a session. Although they reacted quickly to the runway shift event when it occurred in the opposite series position (in SO-r/RE-m or SO-m/RE-r), they clearly reacted more vigorously when it occurred in the original series position (SO-r/RE-r). Thus, this pattern of reactions conforms more to that predicted by the RE or SP hypothesis than to that predicted by the RE at SP hypothesis.

\section{Stay-Win/Shift-Lose Group}

Figure 2B shows that rats in this group slightly but consistently reduced their Trial 3 speeds in the RNR series in each type of test probe. They did not show as consistent an increase in the RNN series, however. Although there was no significant effect of series $[F(1,3)=3.70, p=.15]$, separate analyses revealed that these rats significantly decreased their speeds in the test RNR series $[t(3)=3.56$, $p=.04]$ but did not significantly increase them in the test RNN series $[t(3)=0.39, p=.72]$. These patterns failed to support predictions from the SP-only hypothesis.

Perhaps the relatively small but reliable differences in Trial 3's speeds between the RNR and RNN series during training sessions obscured possible test effects in this group. Another possibility is that our test procedures might have interfered with these rats' ability to determine which series should contain two rewarded trials. If the latter were the case, however, these rats would not have continued to show the Trial 2 speed effect that they had developed during the training phase. Although we did not observe any reduction in such an effect, we analyzed Trial 2 speeds as a function of the series position and type of sessions (odd numbered training sessions vs. even numbered test probe sessions) for each group. We found a significant series effect only for each group $[F \mathrm{~s}(1,3)=9.41 ; 12.85]$. Thus, the rats in each group continued to run reliably less slowly on Trial 2 in a series that would have usually preceded a rewarded Trial 3 than a nonrewarded Trial 3 during the probe test sessions. Therefore, we must attribute our failure to support the SP-only hypothesis in the stay-win/ shift-lose group to a floor effect of speed differences.

\section{EXPERIMENT 2}

As we have already discussed, we designed Experiment 2 to determine whether reversing the predictive validity of each group's specific rewarded Trial 3 runway event would also reverse each group's training and testing effects. New rats were trained under shift-win/stay-lose and stay-win/shift-lose conditions, the only difference being that all rats experienced a runway shift between Trials 1 and 2 in each series. Therefore, given our notation 


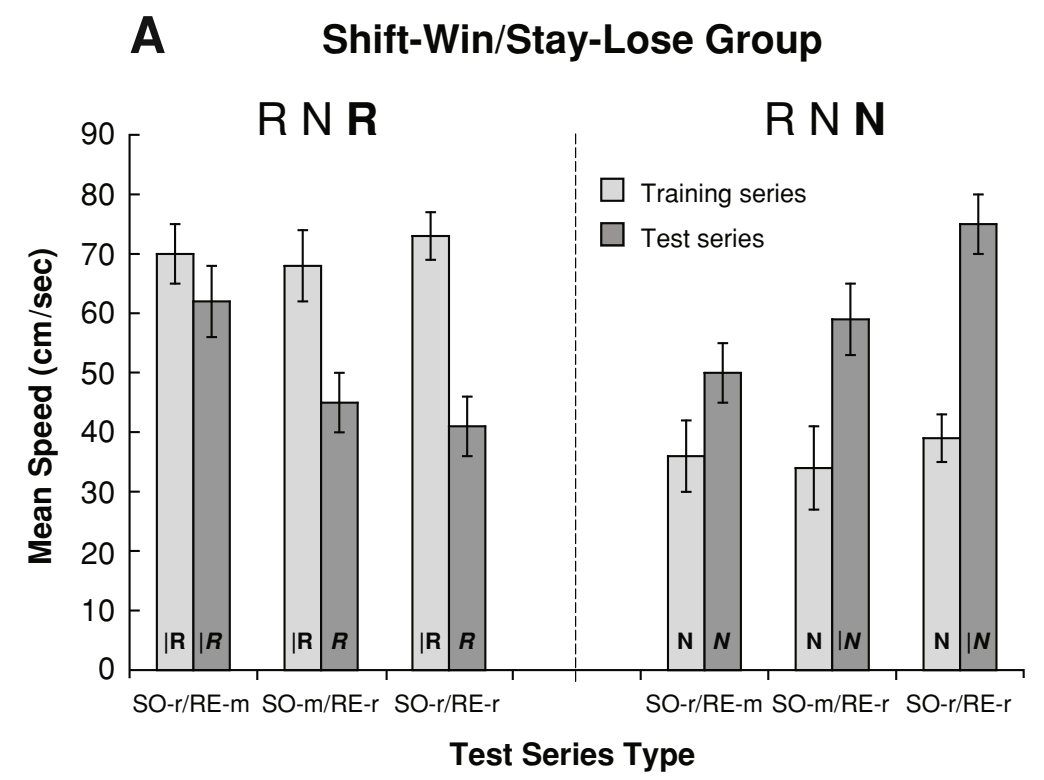

B Stay-Win/Shift-Lose Group

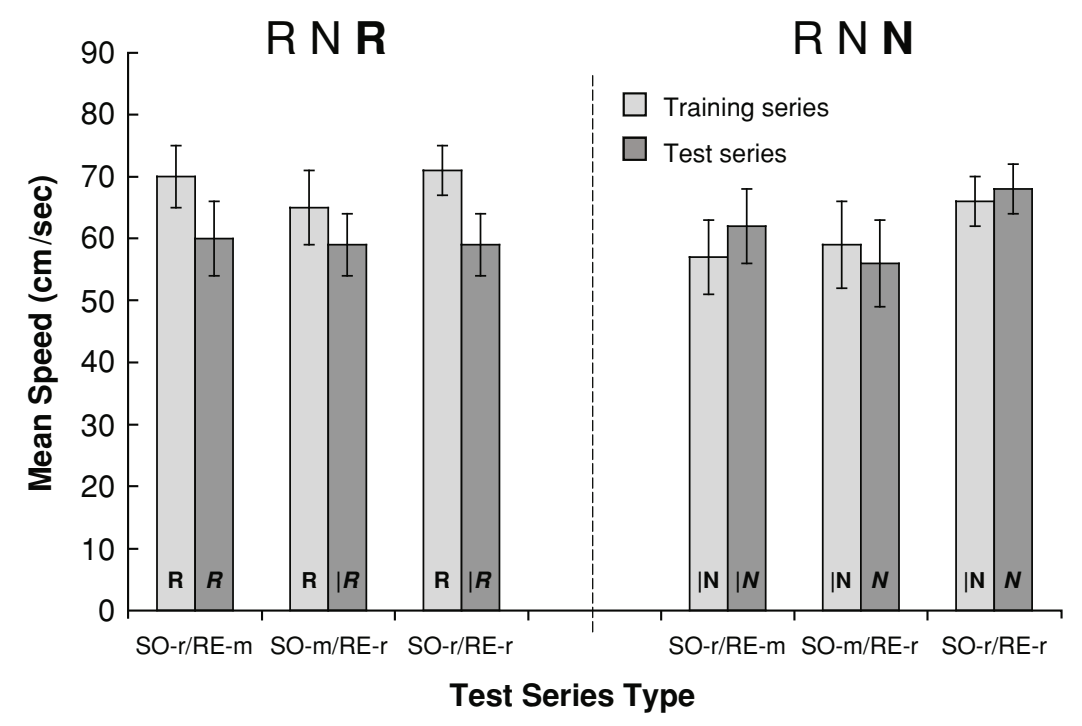

Figure 2. Mean speeds on Trial 3 for each group during the test phase in Experiment 1 for each type of test session and the preceding training sessions. $T$ bars indicate $\pm S E M s$.

in Table 1, the shift-win/stay-lose rats were trained either only on an $R|N| R-R \mid N N$ or only on an $R|N \mathbf{N}-R| N \mid \mathbf{R}$ sequence. The stay-win/shift-lose rats were trained either only on an R|NR-R $|\mathrm{N}| \mathbf{N}$ or only on an $\mathrm{R}|\mathrm{N}| \mathbf{N}-\mathrm{R} \mid \mathrm{NR}$ sequence. Thus, the predictive validity for a Trial 3 reward from a runway shift event was .33 and that for a Trial 3 reward from a runway stay event was 1.00. Following identical extensive training as in Experiment 1, rats received the same test phase with these modified forced choice sequences. From the reversed predictive validities for each Trial 3 runway event, we expected that the stay-win/shift-lose group would learn to determine when Trial 3 was rewarded more easily than would the shiftwin/stay-lose group. Moreover, we expected the stay-win/ shift-lose group's test phase performance to mirror that of the shift-win/stay-lose group in Experiment 1. That is, rats in that group should show that they relied on a runway stay event on Trial 3 or the original position of the RNR series to anticipate a Trial 3 reward (RE or SP hypothesis). We expected that the shift-win/stay-lose group's test phase performance would either mirror that of the staywin/shift-lose group in Experiment 1 or conform more to 
predictions from the SP-only hypothesis. That is, the rats in this group would use RNR position only to anticipate a Trial 3 reward.

\section{Method}

Subjects and Procedure. Twelve naive, 4-month-old male Long-Evans hooded rats purchased from the Charles River Breeding Farms, St. Constant, QC, served in this experiment. They were randomly divided into two equal shift-win/stay-lose and stay-win/ shift-lose groups. We used the same apparatus and procedures as in Experiment 1, except that the rats were exposed to different forced choice patterns in fixed orders as already described.

\section{Results and Discussion}

Training phase. Figure 3 depicts running speeds in the same way as does Figure 1. As in Experiment 1, all rats developed slower speeds on $\mathrm{N}$ trials than on $\mathrm{R}$ trials within each series. Both groups significantly increased their Trial 1 running speeds over blocks of sessions in each series $[F(8,80)=7.18]$. Although each group decreased its always nonrewarded Trial 2 running speeds significantly over blocks $[F(8,80)=24.10]$, the staywin/shift-lose group reduced them more significantly than did the shift-win/stay-lose group in the RNN series $[F(1,10)=7.76]$. Despite a significant overall series effect $[F(1,10)=12.98]$, only the stay-win/shift-lose group developed significantly lower Trial 2 speeds in the RNN series than in the RNR series $[F(1,5)=13.92]$. A similar but smaller difference in the shift-win/stay-lose group was not significant $[F(1,5)=2.26, p=.19]$. The right portion of Figure 3 shows, contrary to predictions, that the shiftwin/stay-lose group developed higher Trial 3 speeds in the RNR series than in the RNN series more quickly than did the stay-win/shift-lose group. Significant interactions of groups $\times$ series $[F(1,10)=45.60]$ and among all three factors $[F(8,80)=3.32]$ confirmed these observations. By the last two blocks of sessions, however, this difference between groups was no longer significant.

The prediction that the stay-win/shift-lose group would perform better than the shift-win/stay-lose group was not confirmed. However, the stay-win/shift-lose group in Experiment 2 seemed to perform better than its counterpart in Experiment 1. That is, rats in this group in Experiment 2 appeared to reduce Trial 2 speeds to lower levels and to develop greater Trial 3 differences in speeds as a function of their outcomes than did the rats in that group in Experiment 1. These differences might be attributed to the runway stay event's having become a more valid predictor for this group. However, making a runway shift event a much less valid predictor for a second reward did not reduce performance in the shift-win/stay-lose group in comparison with that of its counterpart in Experiment 1. The only noticeable difference in this group was that a similar Trial 2 speed effect was not statistically reliable.

Test phase. Figures $4 \mathrm{~A}$ and $4 \mathrm{~B}$ depict the test phase data for this experiment in the same manner as did Figures $2 \mathrm{~A}$ and $2 \mathrm{~B}$ for Experiment 1 . As can be seen, the shift-win/stay-lose group reduced and increased its Trial 3 speeds more on RNR and RNN series, respectively, during probe test sessions than did the stay-win/shift-lose trained group, especially when the series order was maintained but Trial 3 runway events were reversed. A significant effect of group $[F(1,10)=5.64]$ and a significant group $\times$ test probe type interaction $[F(2,20)=6.24]$ supported these observations. Again an $F<1$ for the outcome $\times$ test

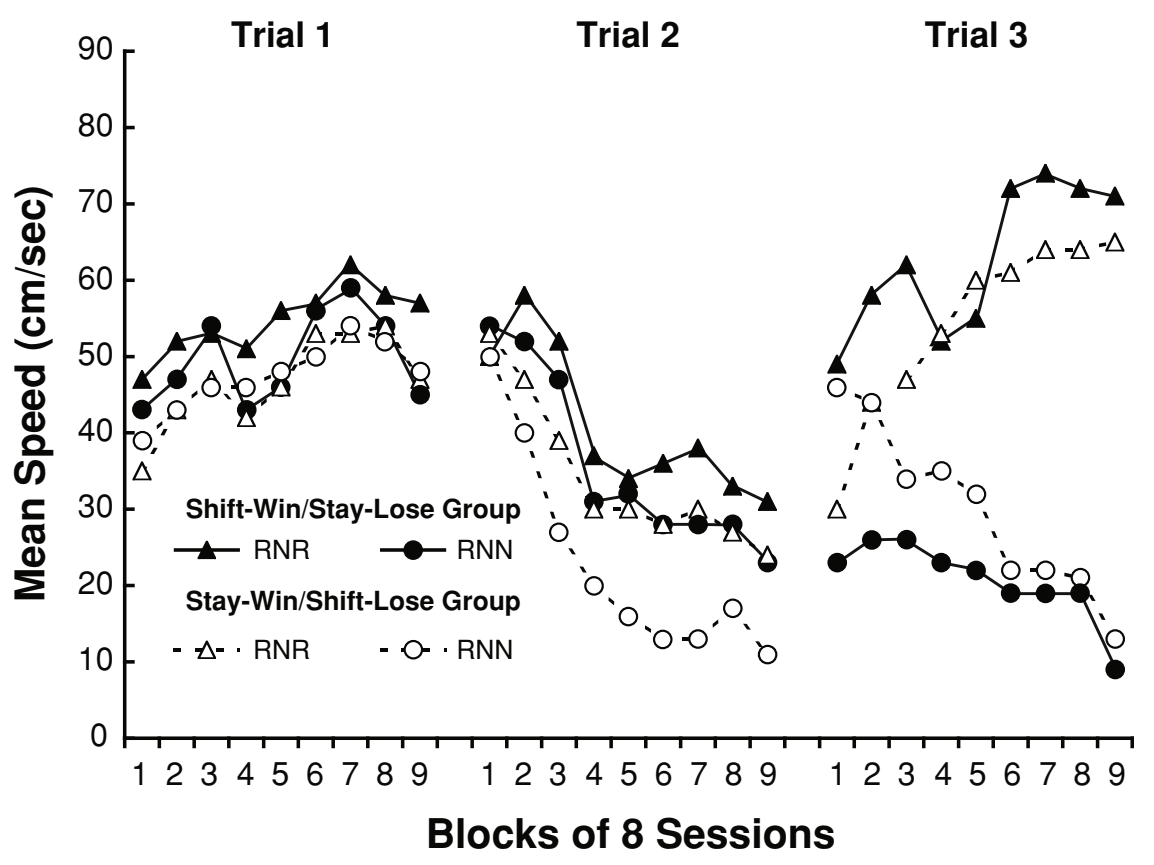

Figure 3. Mean speeds on each trial for each series over blocks of eight sessions for each group during the training phase in Experiment 2. 

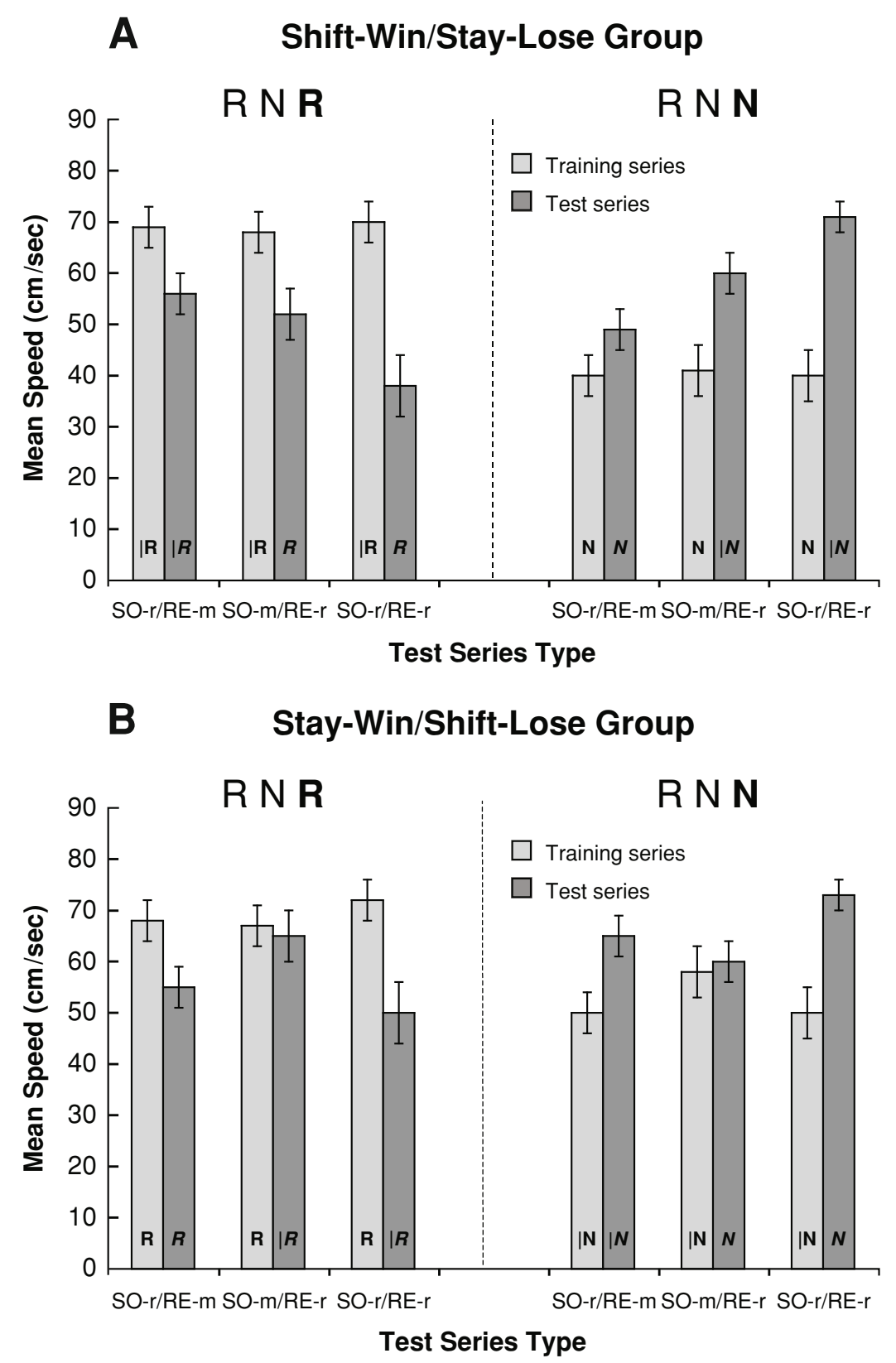

Figure 4. Mean speeds on Trial 3 for each group during the test phase in Experiment 2 for each type of test session and the preceding training sessions. $T$ bars indicate $\pm S E M s$.

session interaction supported the observation of a symmetrical change in speed as a function of trial outcome.

Shift-win/stay-lose group. Figure 4A shows that changes in Trial 3 speeds were greatest when both series order and runway events were reversed (SO-r/RE-r) and lowest when series order was reversed but runway events were maintained (the SO-r/RE-m probe). A significant probe test effect $[F(2,10)=15.92]$ and significant differences between the SO-r/RE-r and either SO-r/RE-m or SO-m/RE-r probe tests $[F \mathrm{~s}(1,5)=26.05 ; 12.10]$ supported this observation. The apparent difference between
SO-m/RE-r and SO-r/RE-m probe tests, however, failed to reach significance $[F(1,5)=5.58, p=.065]$. Consequently, these rats' test performance was very similar to that of their counterparts in Experiment 1. That is, they also evidently learned to rely on the runway shift event and series position separately to determine Trial 3 outcomes and reacted most vigorously when a Trial 3 runway shift occurred in the original series position (SO-r/RE-r).

Stay-win/shift-lose group. As can be seen in Figure 4B, the rats in this group reduced and increased their Trial 3 speeds in the RNR and RNN series, respectively, 
more during SO-r/RE-r and SO-r/RE-m probe tests than during SO-m/RE-r probe tests. Indeed, these rats did not display any consistent changes in Trial 3 speeds in either series in the SO-m/RE-r probe. This observation was supported by a significant probe test effect $[F(2,10)=10.09]$ and pairwise comparisons between SO-m/RE-r and the SO-r/RE-r and SO-r/RE-m tests $\left[F_{\mathrm{S}}(1,5)=41.03 ; 6.70\right]$. Furthermore, changes in Trial 3 speeds within each series were significant in the SO-r/RE-r and SO-r/RE probes $(t \mathrm{~s}>2.50)$ but not in the SO-m/RE-r probes $(t \mathrm{~s}=.48)$. Although speed changes appeared to be slightly greater in the SO-r/RE-r than in the SO-r/RE-m probe tests in each series, these differences were not significant $[F(1,5)=$ $2.10, p=.21]$. These findings suggest that the stay-win/ shift-lose group used series position rather than an equally highly valid runway stay event to anticipate a Trial 3 reward. Thus these results failed to support the prediction that these rats would learn to use their sole runway stay event within the RNR series position to accurately anticipate that trial's reward. Rather, these rats were better able to use RNR series position when a runway stay event occurred only on Trial 3 of that series than when it also occurred on Trial 2 in each series (Experiment 1).

We conducted the same subsidiary statistical analyses as those in Experiment 1 on Trial 2 speeds but did not find any significant effects. The shift-win/say-lose group averaged $33 \mathrm{~cm} / \mathrm{sec}(S E=5.6 \mathrm{~cm} / \mathrm{sec})$ and the stay-win/shiftlose group averaged $28 \mathrm{~cm} / \mathrm{sec}(S E=3.6 \mathrm{~cm} / \mathrm{sec})$. Consequently, the stay-win/shift-lose group could not maintain the reliable differences in Trial 2 speeds as a function of usual Trial 3 outcomes developed during training.

\section{GENERAL DISCUSSION}

In an ordered RNR/RNN serial pattern task, rats learned to run faster on Trial 3 of the RNR series than on Trial 3 of the RNN series more easily when they were forced onto a different runway on that trial only within the RNR series (shift-win/stay-lose group) than only within the RNN series (stay-win/shift-lose group). This difference occurred whether rats were always forced down the same runway on the first two trials in either series (Experiment 1) or down a different runway on Trial 2 from that of Trial 1 in each series (Experiment 2). Posttraining probe tests revealed that the shift-win/stay-lose group in either experiment had learned to rely on the runway shift event in Trial 3 and position of the RNR series within a session to anticipate a reward on Trial 3. The probe tests revealed that the staywin/shift-lose group relied on series position only in Experiment 2. Thus the predictive validity of either type of runway event on Trial 3 for a reward did not influence rats' ability to anticipate that outcome. Reversing the predictive validity of each group's runway event failed to reverse differences in acquisition between groups. It also failed to eliminate the shift-win/stay-lose group's use of a runway shift event or promote the shift-win/stay-lose group's use of a runway stay event for anticipating a reward on Trial 3.
Our findings present problems for understanding serial pattern learning and trial outcome expectancies solely in terms of associative predictive validities. Rats' general predisposition to spontaneously alternate choices in the T-maze (Douglas, 1966) may better account for our results. Such a tendency approximates a general inherent foraging strategy of leaving a depleted patch, a winshift strategy (Charnov, 1976; Krebs \& McCleery, 1984). Therefore, rats may be more prepared to rely on an occasionally rewarded runway shift event than on a more consistently rewarded runway stay event to anticipate a subsequent rewarded trial in a series. Recent findings from our laboratory (Cohen et al., 2004) also show that rats learn to anticipate a second rewarded trial in RRN and RNR series more easily when always forced into the opposite $(\mathrm{R} \mid \mathbf{R N}$ and $\mathrm{RN} \mid \mathbf{R})$ than into the same (RR/N and $\mathrm{R} \mid \mathrm{NR})$ runway for that reward. Thus the shift-win/stay-lose group in our study might not have learned two ordered outcome sequence rules (Hulse, 1980) but applied an inherent foraging choice rule in acquiring this task.

This foraging choice rule idea may allow us to also understand how the stay-win/shift-lose group in Experiment 2 acquired its serial pattern performance more easily than that group in Experiment 1. The rats in this group may not have relied on the highly predictive runway stay event in Experiment 2 because it was contrary to their inherent win-shift foraging strategy. However, they may have used it to learn the series order. The forced choice sequences in this experiment $(\mathrm{R}|\mathrm{N}| \mathrm{N}-\mathrm{R} \mid \mathrm{NR}$ or $\mathrm{R}|\mathrm{NR}-\mathrm{R}| \mathrm{N} \mid \mathrm{N})$ more closely approximate rats' predisposed free choice win-shift spontaneous alternation patterns. Therefore, a forced win-stay departure from this pattern in one of the series (NR sequence) may have served as a redundant highly salient cue for series position. The single runway shift event in Experiment 1 for this group (RN|N-RNR or $\mathrm{RNR}-\mathrm{RN} \mid \mathrm{N}$ ) would not be as salient a series position cue because rats would be predisposed to run into the opposite runway on any subsequent trial anyway. In other words, a sole runway shift event would not be as unexpected or novel for them.

The results from the SO-r/RE-r probe tests suggest that the shift-win/stay-lose groups might have begun to expect a rewarded runway shift event in one series position. However, with such extensive training, these rats might have also begun to form specific running speed patterns (e.g., run fast-slow-slow, then fast-slow-fast; or run fast-slow-fast, then fast-slow-slow). After more training, these groups might have developed more automatic running speed patterns that they would maintain regardless of series order or runway variations on probe tests. Although rats might no longer attend to trial outcomes, series order, or runway variations at that stage, would they also ignore the interseries interval? That is, could they maintain their running speed patterns over the last three trials in the absence of such a temporal phrasing cue? The notion that our rats had started to acquire fixed running speed patterns is based on evidence that rats can acquire a repeated series of fixed free choice sequences in the octagonal free oper- 
ant chamber (Fountain \& Rowan, 1995). In that task, rats obtain reinforcing brain stimulation for pressing levers in a clockwise manner with a counterclockwise choice of a lever after every third correct lever choice (e.g., 123/234/ $345 / 456 / 567 / 678 / 812$, where "/" refers to a series boundary). Later research with this task (Fountain, Benson, \& Wallace, 2000) has indicated that keeping interseries intervals distinctly different from within-series intertrial intervals is necessary for animals to acquire and maintain such choice accuracy.

The present study was not designed to determine whether or to what extent our rats had developed automatic running speed patterns. Our analysis of Trial 2 speeds suggests that they still deliberately processed some information for determining which series contained a second rewarded trial. Both groups in Experiment 1 and the stay-win/shift-lose group in Experiment 2 reliably reduced speeds less on Trial 2 in the RNR series than in the RNN series. Although this effect was maintained during the test phase in Experiment 1, it disappeared in Experiment 2. Capaldi et al. (1983) considered this effect to reflect rats' anticipation of a future trial's outcome during a current trial. That is, they might run slightly less slowly on a second anticipated nonrewarded trial to initiate a more rewarding final trial sooner. Roberts (2002) has questioned whether rats can "time travel" or, in our case, "trial travel." Rather, he suggests that they are "stuck in time" or, in our case, "stuck on the current trial." Indeed, rats cannot be taught to either quickly return to their first entered arm or delay entering a distinctive arm in the eight-arm maze for more food (Roberts \& Roberts, 2002).

The Trial 2 speed effect may indicate that rats are simply uncertain when the second reward will occur, whether on Trial 2 or on Trial 3, in the RNR series. This confusion hypothesis takes two forms. Either rats confuse the ordinal position of Trial 2 with that of Trial 3 (Burns \& Dunkman, 2000; Burns, Wiley, \& Payne, 1986) or they are unable to distinguish between memories of events preceding Trial 2 from those preceding Trial 3-the sequential memory generalization hypothesis (Capaldi \& Miller, 2004). Burns et al. (1986) prevented the Trial 2 speed effect in the ordered RNR/RNN serial pattern task by instituting a longer (10-min) interval between the last two trials in each series. The question remains whether this manipulation made the ordinal position of Trial 3 more distinct from that of Trial 2 or made memories of events preceding Trial 3 distinctly more different from those preceding Trial 2 . In a more recent study (Burns \& Dunkman, 2000), differences in Trial 2 speeds eventually disappeared. Whether this occurred because rats developed better differentiation between trial positions over continued training or developed fixed, automatic running speed patterns is unresolved. Capaldi and Miller found that rats in separate groups ran more slowly on Trial 2 in an RNR series presented with either $\mathrm{N}$ or NN series than in an NNR series presented with R or RN series. The sequential memory model predicts this difference by suggesting that memories of prior outcomes on Trial 2 differ more from those on Trial 3 in the RNR series ( $\mathrm{R}$ vs. $\mathrm{R}+\mathrm{N}$ ) than in the NNR series ( $\mathrm{N}$ vs. $\mathrm{N}+\mathrm{N})$. Perhaps the rats in Experiment 2 of our study did not develop or maintain as consistent a Trial 2 speed effect as did those in Experiment 1 for the same reason. That is, a runway shift on Trial 2 in Experiment 2 generated a more distinctly different memory of events preceding Trial 3 from that of events preceding Trial 2. More systematic research with our preparation is currently under way to test this idea.

\section{REFERENCES}

Burns, R. A., \& Dunkman, J. A. (2000). Ordinal position learning and remote anticipation. Journal of General Psychology, 127, 229-238.

Burns, R. A., Dunkman, J. A., JR., \& Detloff, S. L. (1999). Ordinal position in the serial learning of rats. Animal Learning \& Behavior, 27, 272-279.

Burns, R. A., Wiley, L. P., \& Payne, T. L. (1986). Temporal cuing of runs in series of reward events reduces interevent anticipation. Animal Learning \& Behavior, 14, 190-196.

CAPALDI, E. J. (1992). Levels of organized behavior. In W. K. Honig \& G. Fetterman (Eds.), Cognitive aspects of stimulus control (pp. 385404). Hillsdale, NJ: Erlbaum.

Capaldi, E. J., Birmingham, K. M., \& Miller, R. M. (1999). Forming chunks in instrumental learning: The role of overshadowing. Animal Learning \& Behavior, 27, 221-228.

CAPAldi, E. J., \& Miller, R. M. (2004). Serial learning in rats: A test of three hypotheses. Learning \& Motivation, 35, 71-81.

CAPALDI, E. J., \& Molina, P. (1979). Element discriminability as a determinant of serial-pattern learning. Animal Learning \& Behavior, 7, 318-322.

Capaldi, E. J., NaWrocki, T. M., Miller, D. J., \& Verry, D. R. (1986). Time between events as a retrieval cue: Recall and the temporal similarity between the storage and retrieval intervals. Journal of Experimental Psychology: Animal Behavior Processes, 12, 258-269.

CAPALDI, E. J., NAWrocki, T. M., \& VERry, D. R. (1983). The nature of anticipation: An inter- and intraevent process. Animal Learning \& Behavior, 11, 193-198.

Capaldi, E. J., Verry, D. R., Nawrocki, T. M., \& Miller, D. J. (1984). Serial learning, interitem associations, phrasing cues, interference, overshadowing, chunking, memory, and extinction. Animal Learning \& Behavior, 12, 7-20.

Charnov, E. L. (1976). Optimal foraging, the marginal value theorem. Theoretical Population Biology, 9, 129-136.

Cohen, J. S., Simpson, A., Westlake, K., \& Hamelin, P. (2002). Integration and representation in rats' serial pattern learning in the T-maze. Animal Learning \& Behavior, 30, 261-274.

Cohen, J. S., Westlake, K., \& Pepin, M. (2001). Higher order chunking in serial pattern learning by rats in the T-maze. Learning \& Motivation, 32, 408-433.

Cohen, J. [S.], Westlake, K., \& Szelest, I. (2004). Effects of runway shift and stay rules on rats' serial pattern learning in the T-maze. Learning \& Behavior, 32, 500-511.

Douglas, R. J. (1966). Cues for spontaneous alternation. Journal of Comparative \& Physiological Psychology, 62, 171-183.

Fountain, S. B., Benson, A. M., \& Wallace, D. G. (2000). Number, but not rhythmicity, of temporal cues determines phrasing effects in rat serial-pattern learning. Learning \& Motivation, 31, 301-322.

Fountain, S. B., Henne, D. R., \& Hulse, S. H. (1984). Phrasing cues and hierarchical organization in serial pattern learning by rats. Journal of Experimental Psychology: Animal Behavior Processes, 10, 30-45.

Fountain, S. B., \& Rowan, J. D. (1995). Coding of hierarchical versus linear pattern structure in rats and humans. Journal of Experimental Psychology: Animal Behavior Processes, 21, 187-202.

Haggbloom, S. J. (1993). Positive transfer across grouping cue modalities in rat serial learning. Learning \& Motivation, 24, 266-281.

Haggbloom, S. J., Birmingham, K. M., \& Scranton, D. L. (1992). Hierarchical organization of series information by rats: Series chunks and list chunks. Learning \& Motivation, 23, 183-199. 
Hulse, S. H. (1978). Cognitive structure and serial pattern learning by animals. In S. H. Hulse, H. Fowler, \& W. K. Honig (Eds.), Cognitive processes in animal behavior (pp. 311-340). Hillsdale, NJ: Erlbaum.

Hulse, S. H. (1980). The case of the missing rule: Memory for reward vs. formal structure in serial-pattern learning by rats. Animal Learning \& Behavior, 8, 689-690.

Krebs, J. R., \& McCleery, R. H. (1984). Optimization in behavioral ecology. In J. R. Krebs \& N. B. Davies (Eds.), Behavioral ecology (2nd ed., pp. 91-121). Sunderland, MA: Sinauer.

Miller, G. A. (1956). The magic number seven plus or minus two:
Some limits to our perspective for processing information. Psychological Review, 63, 81-87.

Roberts, W. A. (2002). Are animals stuck in time? Psychological Bulletin, 128, 473-489.

Roberts, W. A., \& Roberts, S. (2002). Two tests of the stuck-in-time hypothesis. Journal of General Psychology, 129, 415-429.

Simon, H. A. (1974). How big is a chunk? Science, 183, 482-488.

(Manuscript received May 18, 2005;

revision accepted for publication November 7, 2005.) 